\title{
LUPUS SKIN FLARE AFTER USE OF CAPILLARY PATCH
}

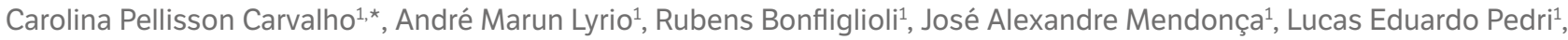
Flávia Regina Andrade ${ }^{1}$, Fernanda Bertucci Sanches ${ }^{1}$, Cláudia Valeria Vierhout ${ }^{1}$, Marina de Sousa Vieira ${ }^{1}$, William Barros Hyppolito Ferreira ${ }^{1}$, Vanessa Félix Nascimento Coelho ${ }^{1}$, Maria Veronica Russo Macchi ${ }^{1}$, Nayara Mota Carvalho ${ }^{1}$

1.Pontifícia Universidade Católica de Campinas, Campinas (SP), Brazil.

*Corresponding author: carolpellisson@hotmail.com

\section{BACKGROUND}

Systemic lupus erythematosus (SLE) is a disease of multifactorial etiology, chronic inflammatory, autoimmune, more common in women, with interspersed periods of exacerbation and remission. It presents cutaneous, joint, cardiac, pulmonary, neuropsychiatric, renal and hematological manifestations, with the occurrence of cutaneous involvement in more than $75 \%$ of cases. Discoid lupus erythematosus (LE) is the most common subtype of chronic cutaneous LE, characterized by erythematous, infiltrated plaques with central atrophy and peripheral dyschromia, mainly in the head and neck.

\section{CASE REPORT}

Female patient, 32 years old, diagnosed with lupus nephritis during pregnancy 6 years ago and needing dialysis for 3 months, developing alopecia, in addition to reactive anti-DNA and anti-SM, mixed ANA 1:640, featuring LES. The patient makes regular use of hydroxychloroquine $400 \mathrm{mg} /$ day, prednisone $40 \mathrm{mg} /$ day, methotrexate $12.5 \mathrm{mg} /$ week and an antiproteinuric agent, presented erythematous scaly lesions on the scalp after the use of capillary patch, which progressed to the cervix and upper limbs, refractory to increased oral and topical corticosteroid therapy. Due to the emergence of new lesions with infiltrated erythematous borders and an atrophic center in the nostril, arms and scalp, hospitalization and pulse therapy with methylprednisolone for 3 days and methotrexate $25 \mathrm{mg}$ intravenously was chosen, maintaining the antimalarial drug. The patient showed significant improvement in the lesions, being weaned from corticosteroids via the outpatient clinic, increased hydroxychloroquine to twice a day due to obesity, and methotrexate $25 \mathrm{mg} /$ week was maintained.

\section{CONCLUSION}

The most frequent SLE manifestation are the skin lesions, and in the worse cases of the disease, pulse therapy and methylprednisolone can be used together for 3 days and an immunosuppressant, besides from the antimalarial drug, as in the reported case, presenting a positive result in regards to the improvement of the skin case.

\section{KEYWORDS}

Discoid lupus erythematosus, Capillary patch, Pulse therapy. 